\title{
Hydroboration with Pyridine Borane
}

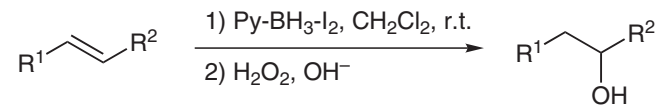

$R^{1}=$ Me, $R^{2}=P h: \quad 92 \%$ yield, 15:1 regioselectivity

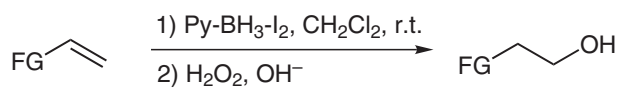

$\mathrm{FG}=\mathrm{OBz}, \mathrm{NBn}_{2}, \mathrm{NHBz} \quad 74-98 \%$ yield, $>19: 1$ regioselectivity
Significance: A stable available pyridine-borane complex after the reaction with iodine gives a selective hydroborating agent, active at room temperature. Acetylenes can be transformed into ketones after oxidation. Mono-, di- and trisubstituted alkenes can be readily hydroborated leading to mono-adducts at room temperature. Functional groups like esters, amines and amides are tolerated.
Comment: Despite the numerous existing hydroborating agents, developed to date, there is still the lack of a cheap, selective, robust and stable reagent. Pyridine-borane complex is a stable and not air-sensitive liquid. On the reaction with iodine, it is transformed into a strong but selective hydroborating agent, that reacts equally well with alkynes and alkenes, tolerating reducible functional groups. The procedure is very simple and practical. 